\title{
By chance, or by design?
}

\section{Enzymes are well known for speeding up reactions. But have they evolved to use quantum mechanics to exert their effects? Philip Ball meets the researchers who are trying to find out.}

$\mathrm{M}$ ost biologists would scoff at the idea that their subject is simply applied quantum mechanics. But for some enzymes - the catalysts of biology - quantum effects may be an important part of the way they work. This revelation has left chemists and biologists arguing about whether enzymes have evolved to do this, or whether the effect would happen regardless of the enzymes' activity.

At the heart of this debate is the issue of how much control enzymes have over the processes that affect their catalytic power. It's a debate about the limits of molecular biology's capabilities and inventiveness. For researchers trying to design new enzymes for chemical and biochemical synthesis, the idea that natural enzymes can manipulate quantum effects to their advantage could suggest new possibilities. At the same time, it could make the design process a lot more complex.

The effect in question is known as quantum tunnelling. This offers quantum objects — such as a hydrogen atom - a way to cross an insurmountable energy barrier that they cannot get over 'classically'. Rather than needing sufficient energy to overcome the barrier, the object just tunnels through it instead. "There is now ample evidence that tunnelling occurs in enzymes, and the biochemical community generally accepts
"Tunnelling is a fact of life, but life has no special effect on tunnelling."

\section{— Willem Siebrand}

so there is always a small chance that the particle will appear on the other side of a barrier.

The probability that quantum tunnelling will occur falls as the object gets heavier, which is why it never actually happens to a marble. In fact, it is unlikely to occur for most atoms in chemical reactions - unless they are as small and light as a hydrogen atom. "Hydrogen transfer is probably one of the most fundamental and prevalent processes in biology," says Judith Klinman, a protein chemist at the University of California, Berkeley, who has championed the case for tunnelling in enzymes since the late 1980s.

\section{Digging in}

The possibility that hydrogen tunnelling occurs in biochemical reactions was first raised over 30 years ago. But it wasn't until 1989 that Klinman and her colleagues reported direct evidence for it ${ }^{1}$. They studied a yeast enzyme called alcohol dehydrogenase $(\mathrm{ADH})$, which transfers a hydrogen atom from an alcohol to a small molecule known as nicotinamide adenine dinucleotide.

To test for tunnelling, the team looked for the kinetic isotope effect (KIE) a small change in the rate of the enzyme's reaction. If the hydrogen atom to be moved from the alcohol is switched for a heavier isotope - deuterium or tritium - the bond energy changes, this to be the case," says Nigel Scrutton, a biochemist at the University of Leicester, UK. In fact, tunnelling can speed up a reaction by a thousand times or more, he adds.

But the question of whether enzymes have evolved to make the most of quantum tunnelling has provoked a heated reaction. Not everyone agrees that molecular biology is smart enough to have such an effect.

"This is a big red herring," says Arieh Warshel, a theoretical biochemist at the University of Southern California. The critics say that quantum tunnelling happens regardless of what the enzyme does, and has nothing to do with evolutionary fine-tuning.

In classical terms, quantum tunnelling is like a marble inside a jar vanishing and then reappearing outside the jar. It seems impossible but, in the quantum world, it can happen because all quantum particles have wave-like properties. This means that their location is defined by a varying probability over space- which in turn alters the reaction rate. Classically, it is fairly easy to predict what this change should be. But if tunnelling is involved, then there is an additional effect on the rate. So a KIE with properties different from the one predicted classically indicates that quantum tunnelling has taken place. This is exactly what Klinman and her team saw.

Since then, they and others have found evidence for tunnelling in several enzymatic reactions ${ }^{2,3}$. Klinman now thinks that enzymes can enhance hydrogen tunnelling to increase their reaction rates. Working with chemists in Italy in 1999, she found that some enzymes seem to adjust the amount of tunnelling to suit their operating temperature ${ }^{4}$.

This time, the team was looking at an $\mathrm{ADH}$ from the thermophilic bacterium Bacillus stearothermophilus. This enzyme works at a temperature of $65^{\circ} \mathrm{C}$, some $40{ }^{\circ} \mathrm{C}$ hotter than the yeast equivalent. Tunnelling is expected to make less of a contribution to the
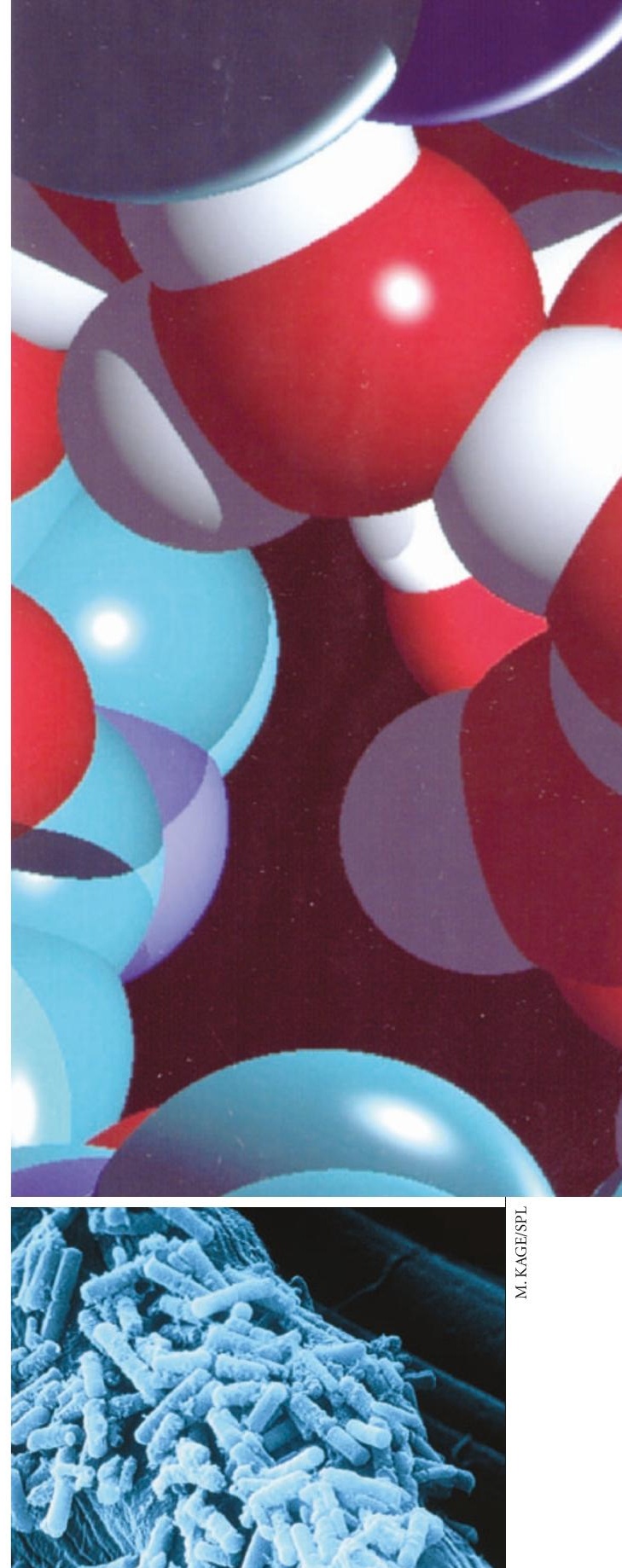

Bacillus stearothermophilus uses an enzyme that seems to adapt to its working temperature.

reaction rate for enzymes operating at higher temperatures. But the researchers found that the tunnelling contribution was similar for both the yeast and the thermophilic $\mathrm{ADH}$.

\section{Tunnel vision}

Others argue that tunnelling occurs irrespective of what the enzyme is doing. "All hydrogen reactions involve some degree of tunnelling, depending on the temperature," says Richard Finke, a chemist at Colorado State University in Fort Collins. So perhaps biology isn't 'using' this quantum effect, but simply failing to do anything either to prevent or to enhance it.

Finke says that a proper test of the hypothesis would mean comparing the amount of tunnelling that occurs in the pres- 


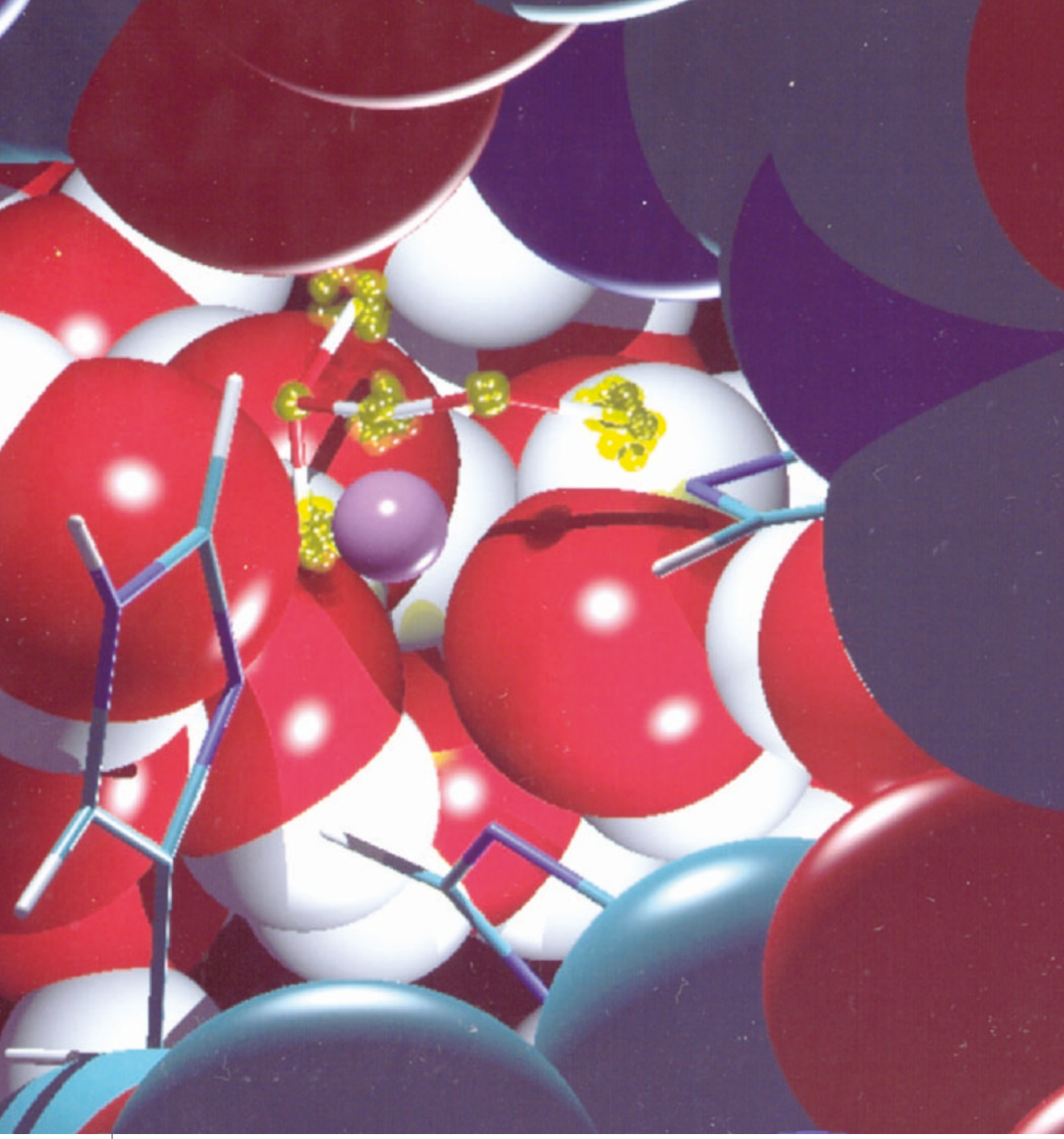

Models of biocatalysis suggest that quantum tunnelling occurs whether or not an enzyme is present.

ence and absence of the enzyme. But this is difficult to do, because in most cases the reaction won't proceed without an enzyme.

In 2000, Finke found the test he was looking for. The enzyme methylmalonyl-CoA mutase catalyses a hydrogen-transfer reaction but requires a cofactor, a molecule called AdoCbl, for the reaction to proceed. The enzyme breaks a cobalt-carbon bond in AdoCbl to generate a free radical, which then pulls a hydrogen atom off another molecule (methyl malonate-CoA). Previously, this reaction had shown evidence of tunnelling.

But a very similar reaction occurs without the enzyme if AdoCbl is heated in ethylene glycol solution. In the enzyme-free reaction, the ethylene glycol, rather than methyl malonateCoA, provides the hydrogen atom. But Finke and his colleagues figured that the reaction rate should be largely unaffected by these different sources of hydrogen, so that the extent of tunnelling with or without the enzyme could be directly compared.

They found that the tunnelling rates were more or less identical, within experimental error $^{5,6}$. In other words, there is no reason to believe that the enzyme is doing anything special. Such results have persuaded Willem
Siebrand, a theoretical chemist at the Steacie Institute for Molecular Sciences in Ottawa, Canada, that "tunnelling is a fact of life, but life has no special effect on tunnelling".

But, as Finke points out, the evolutionary pressure on the enzyme to take advantage of quantum-mechanical tunnelling is weak in this case. This is because the enzyme's main job is to break the cobalt-carbon bond in AdoCbl, rather than to pluck out a hydrogen atom. The different hydrogen sources for the two reactions also worry Klinman and Scrutton. "It is very dangerous to generalize on the basis of one comparison of model chemistry with enzymatic chemistry," says Scrutton.

Warshel argues that because it is so hard to compare catalysed and uncatalysed reactions experimentally, it is better to use computer simulations. In 1996, he and Jenn-Kang Hwang from National Tsing Hua and prevalent processes in biology."

University in Taiwan simulated the behaviour of the enzyme carbonic anhydrase and incorporated quantum effects such as tunnelling ${ }^{7}$. They compared their model with one for the same process happening in an enzyme-free solution, and found a similar amount of tunnelling in both cases.

It is much harder than some people think for enzymes to manipulate their reactants to optimize the amount of tunnelling, says Warshel. To do this, they would have to change the shape of the energy barrier that separates the reactants from the products. Warshel thinks that enzymes are too floppy for that. "The idea that enzymes can do 'anything' is wrong," he says.

But those who believe that enzymes can make use of quantum tunnelling argue that the proteins could use dynamic behavioursuch as their molecular vibrations - to 'squeeze' the hydrogen through the energy barrier. Scrutton admits that this idea remains controversial, however.

\section{Good vibrations}

Klinman claims that an enzyme's vibrations can be used to make tunnelling easier - for example, by reducing the distance over which the hydrogen has to tunnel between the source and the target molecules, or by altering the relative energy levels of the reactants and products. In theoretical calculations ${ }^{8}$, the hydrogen-tunnelling distance in a reaction catalysed by the enzyme soybean lipoxygenase seems to be shorter than the distance between the source and target molecules. This suggests that vibrations are needed to bring the two molecules closer together when tunnelling occurs.

Klinman is convinced that evolution can select particular vibrational states to enhance enzyme catalysis. Scrutton, meanwhile, believes that even if vibration-assisted tunnelling does occur, it is not absolutely essential. In his view, the available evidence suggests that many enzymes are already optimized for tunnelling without the help of vibrations. For example, his group has found that the enzyme trimethylamine dehydrogenase doesn't seem to exploit vibrations in its natural form but does make use of them in a mutant form ${ }^{9}$.

Others dispute the whole idea. Siebrand thinks that "proteins are far too flexible to be good squeezers", and that the process would require too much energy. Warshel agrees. "On balance there is no evidence for dynamical contributions to catalysis," he says.

The debate shows little sign of being resolved quickly. And until it is, we must remain uncertain about the limits of nature's ingenuity.

\section{Philip Ball is a consultant editor for Nature.}

1. Cha, Y., Murray, C. J. \& Klinman, J. P. Science 243, 1325-1330 (1989).

2. Kohen, A. \& Klinman, J. P. Acc. Chem. Res. 31, 397-404 (1998).

3. Sutcliffe, M. J. \& Scrutton, N. S. Eur. J. Biochem. 269, 3096-3102 (2002)

4. Kohen, A., Cannio, R., Bartolucci, S. \& Klinman, J. P. Nature 399, 496-499 (1999).

5. Doll, K. M., Bender, B. R. \& Finke, R. G. J. Am. Chem. Soc. $\mathbf{1 2 5}$ 10877-10884 (2003).

6. Doll, K. M. \& Finke, R. G. Inorg. Chem. 42, 4849-4856 (2003).

7. Hwang, J.-K. \& Warshel, A. J. Am. Chem. Soc. 118, 11745-1175 (1996).

8. Hatcher, E., Soudackov, A. V. \& Hammes-Schiffer, S. J. Am. Chem. Soc. 126, 5763-5775 (2004).

9. Basran, J., Sutcliffe, M. J. \& Scrutton, N. S. J. Biol. Chem. 276, 24581-24587 (2001) 